\section{(C) OPEN ACCESS}

\title{
HIV and herpes simplex virus type 2 epidemiological synergy: misguided observational evidence? A modelling study
}

\author{
Ryosuke Omori, ${ }^{1,2}$ Nico Nagelkerke, ${ }^{3,4,5}$ Laith J Abu-Raddad ${ }^{6,7}$
}

\begin{abstract}
${ }^{1}$ Division of Bioinformatics, Research Center for Zoonosis Control, Hokkaido University, Sapporo, Japan ${ }^{2}$ PRESTO, JST, Kawaguchi, Saitama, Japan

${ }^{3}$ Department of Public Health, Erasmus MC, University Medical Center Rotterdam, Rotterdam, The Netherlands

${ }^{4}$ Medical Microbiology, University of Manitoba, Winnipeg, Canada ${ }^{5}$ Institute of Public Health, College of Medicine and Health Science, United Arab Emirates University, Al Ain, United Arab Emirates

${ }^{6}$ Infectious Disease

Epidemiology Group, Weill Cornell Medicine-Qatar, Cornell University, Doha, Qatar ${ }^{7}$ Department of Healthcare Policy and Research, Weill Cornell Medicine, Cornell University, New York, USA
\end{abstract}

\section{Correspondence to}

Dr Ryosuke Omori, Division of Bioinformatics, Research Center for Zoonosis Control, Hokkaido University, Sapporo 001-0020, Japan; omori@czc.hokudai.ac.jp

Received 4 July 2017 Revised 24 October 2017 Accepted 4 November 2017 Published Online First 4 December 2017
Check for updates

To cite: Omori $\mathrm{R}$, Nagelkerke N, AbuRaddad LJ. Sex Transm Infect 2018:94:372-376.

\begin{abstract}
Objectives To investigate whether observational studies of HIV and herpes simplex virus type 2 (HSV-2) infections have the capacity to assess the HIV/HSV-2 epidemiological synergy.

Methods An individual-based Monte Carlo model was used to simulate HIV/HSV-2 epidemics in two scenarios: no HIV/HSV-2 biological interaction and HSV-2 seropositivity enhancing HIV acquisition. Cross-sectional observational studies were simulated by sampling individuals from the population to assess resulting crude and adjusted ORs of the HIV/HSV-2 association. Metaanalyses were conducted to estimate the pooled mean ORs. Impact of under-reporting of sexual behaviour and miscapture of high-risk individuals was assessed through sensitivity analyses.
\end{abstract}

Results Assuming no HIV/HSV-2 biological interaction, the crude HIV/HSV-2 OR ranged between 1.38 and 9.93, with a pooled mean of 6.45 ( $95 \% \mathrm{Cl} 5.81$ to 7.17$)$. Adjustment for the number of sexual partners over last year, over lifetime and for both partner numbers simultaneously reduced the mean OR to $5.45(95 \% \mathrm{Cl}$ 4.90 to 6.06$), 3.70(95 \% \mathrm{Cl} 3.32$ to 4.12$)$ and 3.54 (95\% Cl 3.17 to 3.94 ), respectively. Assuming HIV/HSV2 biological interaction, the crude OR ranged between 3.44 and 9.95 , with a pooled mean of $8.05(95 \% \mathrm{Cl}$ 7.14 to 9.07$)$. The adjustments reduced the mean OR to 7.00 (95\% Cl 6.21 to 7.90$), 3.76(95 \% \mathrm{Cl} 3.32$ to 4.25$)$ and $3.68(95 \% \mathrm{Cl} 3.25$ to 4.17$)$, respectively. Underreporting of partners reduced the confounder-adjustment effects. Miscapture of high-risk individuals considerably lowered the estimated ORs.

Conclusions It is difficult to control for sexualbehaviour confounding in observational studies. The observed HIV/HSV-2 association appears more consistent with two infections sharing the same mode of transmission, rather than with HSV-2 enhancing HIV acquisition.

\section{INTRODUCTION}

The biological interactions and epidemiological synergy between HIV and herpes simplex virus type 2 (HSV-2) infections have been intensely investigated. ${ }^{1}$ There is ample observational evidence for a strong HIV/HSV-2 association, and that HSV-2 enhances HIV acquisition. ${ }^{2-4}$ Mathematical models incorporating this synergy estimated that HSV-2 has been a major contributor to the HIV epidemic in sub-Saharan Africa (SSA). ${ }^{15}$
Nevertheless, HIV and HSV-2 share the same sexual mode of transmission and propagate on the same sexual networks. It is 'natural' for these two infections to be strongly associated regardless of any HIV/HSV-2 biological interaction. Observational studies attempted to control for this confounding factor by adjusting the measured associations for sexual behaviour, often by controlling for the number of self-reported sexual partners. ${ }^{4}$ As adjusted associations were still positive and strong, this supported the existence of a biological interaction, and consequently an epidemiological synergy, between these two infections. ${ }^{4}$ As most self-reported sexual data are egocentric, network effects could not be adjusted for in these observational studies.

The difficulty of controlling for network effects, however, limits the validity of these observational studies. The risk of acquiring an STI depends not only on a person's behaviour, but also on the "ecology" of the person within the sexual network, including the partners and partners' partners behaviour, past and present. ${ }^{6-11}$ Sexual networks, therefore, play a critical role in determining STI patterns. ${ }^{6812}$ For example, it has been hypothesised that concurrency is a main driver of the generalised HIV epidemics in SSA. ${ }^{13-15}$

Several clinical trials were conducted to target the HIV/HSV-2 epidemiological synergy, by suppressing HSV-2 reactivation to reduce HIV acquisition and transmission, but these trials reached negative outcomes. ${ }^{16-18}$ Different explanations have been advanced for these negative results, such as ineffective HSV-2 suppression, insufficient dose or therapy duration, and poor adherence. ${ }^{16-19}$ It is natural, however, to also question the very existence of this epidemiological synergy - that is, the observed HIV/HSV-2 association could be a spurious association not reflecting a genuine biological effect; it arose from intrinsic biases in study design due to failure to adequately control for confounding by sexual behaviour.

Here we investigate two related questions: Can observational studies adequately control for confounding by sexual behaviour? Is the observed association consistent with an absence of epidemiological synergy? We addressed these two questions through mathematical modelling by first simulating HIV/HSV-2 transmission in diverse sexual networks assuming no HIV/HSV-2 biological interaction. We 
then simulated HIV/HSV-2 transmission assuming HIV/HSV-2 biological interaction with an effect size as that reported in observational studies. ${ }^{2-4}$ Subsequently, we simulated the conduct and analysis of observational studies on the simulated epidemics, that is, we cross-sectionally sampled individuals from the population and estimated sample associations by logistic regressions.

In light of known limitations to observational evidence, namely under-reporting of sexual behaviour and miscapture (undersampling) of high-risk individuals in surveys, we repeated our analyses factoring in these limitations. Our study thus furnishes a 'microscopic' assessment, from first principles, of one of the most controversial factors in HIV epidemiology.

\section{METHODOLOGY}

We used a recently constructed individual-based Monte Carlo simulation model $^{6}$ to simulate sex partnering, birth/death and HIV/HSV-2 transmission in a population. The model was applied in two scenarios. First, HIV/HSV-2 transmission was simulated assuming no HIV/HSV-2 biological interaction. Second, HIV/HSV-2 transmission was simulated assuming that HSV-2 seropositivity increases the risk of HIV acquisition by 2.5 -foldthe relative risk effect size as estimated from observational studies. $^{2-4}$ In the latter scenario, we lowered the overall average level of sexual risk behaviour so that both scenarios had similar HIV prevalence levels. Accordingly, the second scenario can be seen as a 'positive control' of the first scenario.

The model was parameterised with representative natural history and transmission parameters, ${ }^{16}$ listed in online supplementary table S1. Since observational studies suggest that the effect size of the HIV/HSV-2 association varies between populations, such as the general population versus high-risk populations, ${ }^{3}$ we assessed the association in diverse sexual networks to accommodate for potential variability in effect size by type of population/sexual network.

The sexual networks were simulated based on the range of population-based sexual behaviour data in SSA, ${ }^{20}$ as derived from 25 Demographic and Health Surveys (DHS) data. ${ }^{21}$ Marital partnerships formed/dissolved at a rate derived from DHS current-marriage age-specific data. ${ }^{6}{ }^{21}$ Non-marital partnerships formed/dissolved in unmarried (premarital sex) and married (extramarital sex) persons, but at different DHS-derived rates. ${ }^{620}$ Sexual behaviour parameters were sampled (randomly) from the empirical distributions ${ }^{20}$ to generate a diversity of sexual network statistics such as number-of-partners mean and variance, degree correlation, clustering coefficient, and concurrency-accommodating for variation by marital status and age. ${ }^{6}$

We simulated $100 \mathrm{HIV} / \mathrm{HSV}-2$ epidemics on 100 different sexual networks, with initial population sizes of 2000, for each scenario-numbers sufficient to be representative of the diversity of the (stochastic) epidemics we see for these two infections. Each simulation was seeded with $10 \mathrm{HIV} / \mathrm{HSV}-2$ infections, and was run with a 'burn-in' time of 200 years to reach equilibrium. Although the model allows assessment of the HIV/HSV-2 association temporally and by epidemic phase, we only assessed the association at endemic equilibrium to control for potential temporal effects on the association.

We cross-sectionally sampled all individuals 15-65 years of age from each of the 100 simulated epidemics/populations. In each sample, we assessed the HIV/HSV-2 association. Different univariable and multivariable logistic regression analyses were conducted to calculate crude and adjusted ORs of HIV infection with HSV-2 seropositivity. The following independent predictors were selected a priori because of relevance to the research questions: number of sexual partners over the last year (continuous variable), number of lifetime partners (continuous variable), age (continuous variable) and marriage (categorical variable), in addition to HSV-2 serostatus (categorical variable). Different regression models were used to adjust for the number of sexual partners over the last year, number of lifetime partners, both numbers of partners, and both numbers of partners, age and marriage. The latter analysis was done to reflect common adjustment variables in observational studies. ${ }^{4}$ ORs of the 100 simulated studies were pooled using a DerSimonian-Laird random-effects model to calculate pooled effects.

For sensitivity analyses, we repeated the above analyses for each scenario, but after reducing (randomly) the reported number of partners over the last year, for individuals with $>1$ partners, by an average of $10 \%, 25 \%$ and $50 \%$, to simulate under-reporting of sexual behaviour. We also repeated the above analyses, but after excluding individuals with the highest $10 \%$, $25 \%$ and $50 \%$ number of partners over the last year, to simulate miscapture of high-risk individuals.

\section{RESULTS}

Table 1 shows the results of the regression analyses assuming no HIV/HSV-2 biological interaction. In the baseline scenario of no under-reporting and no miscapture, the crude OR ranged between 1.38 and 9.93, with a pooled mean of 6.45 (95\% CI 5.81 to 7.17$)$. Adjustment for the number of partners over last year, over lifetime and for both numbers of partners reduced the mean OR to 5.45 (95\% CI 4.90 to 6.06), 3.70 (95\% CI 3.32 to 4.12) and 3.54 (95\% CI 3.17 to 3.94), respectively. Adjustment for age and marriage in addition to partner numbers yielded an OR of 4.93 (95\% CI 4.41 to 5.52). The sensitivity analyses assuming under-reporting and miscapture yielded similar patterns.

Table 2 shows the results of the regression analyses assuming increased HIV acquisition with HSV-2 seropositivity. In the baseline scenario of no under-reporting and no miscapture, the crude OR was higher with a range of 3.44-9.95 and a pooled mean of 8.05 (95\% CI 7.14 to 9.07). Adjustment for the number of partners over last year, over lifetime and for both numbers of partners reduced the mean OR by larger margins than those with no HIV/HSV-2 biological interaction-the mean OR was reduced to 7.00 (95\% CI 6.21 to 7.90$), 3.76$ (95\% CI 3.32 to 4.25$)$ and 3.68 (95\% CI 3.25 to 4.17), respectively. Adjustment for age and marriage in addition to partner numbers yielded an OR of 4.71 (95\% CI 4.15 to 5.35). The sensitivity analyses assuming under-reporting and miscapture yielded similar patterns.

Adjustments for the lifetime number of partners always had larger effects on the OR than those for the last-year number of partners (tables 1 and 2). The simultaneous adjustment for both numbers of partners resulted in a larger effect on the OR, although not much larger than that for only the lifetime number of partners. Under-reporting of partners reduced the adjustment effects for both numbers of partners. Miscapture of high-risk individuals considerably lowered the estimated ORs.

\section{DISCUSSION}

Our results demonstrate the difficulty of adequately controlling for sexual-behaviour confounding. While the ORs were closer to 1 after adjustment for numbers of partners, they remained well above 1 despite absence of any biological interaction in the first modelling scenario. The lifetime-partner-number adjustment was also more effective, probably because it captured better the exposure risk to both infections over lifetime. 


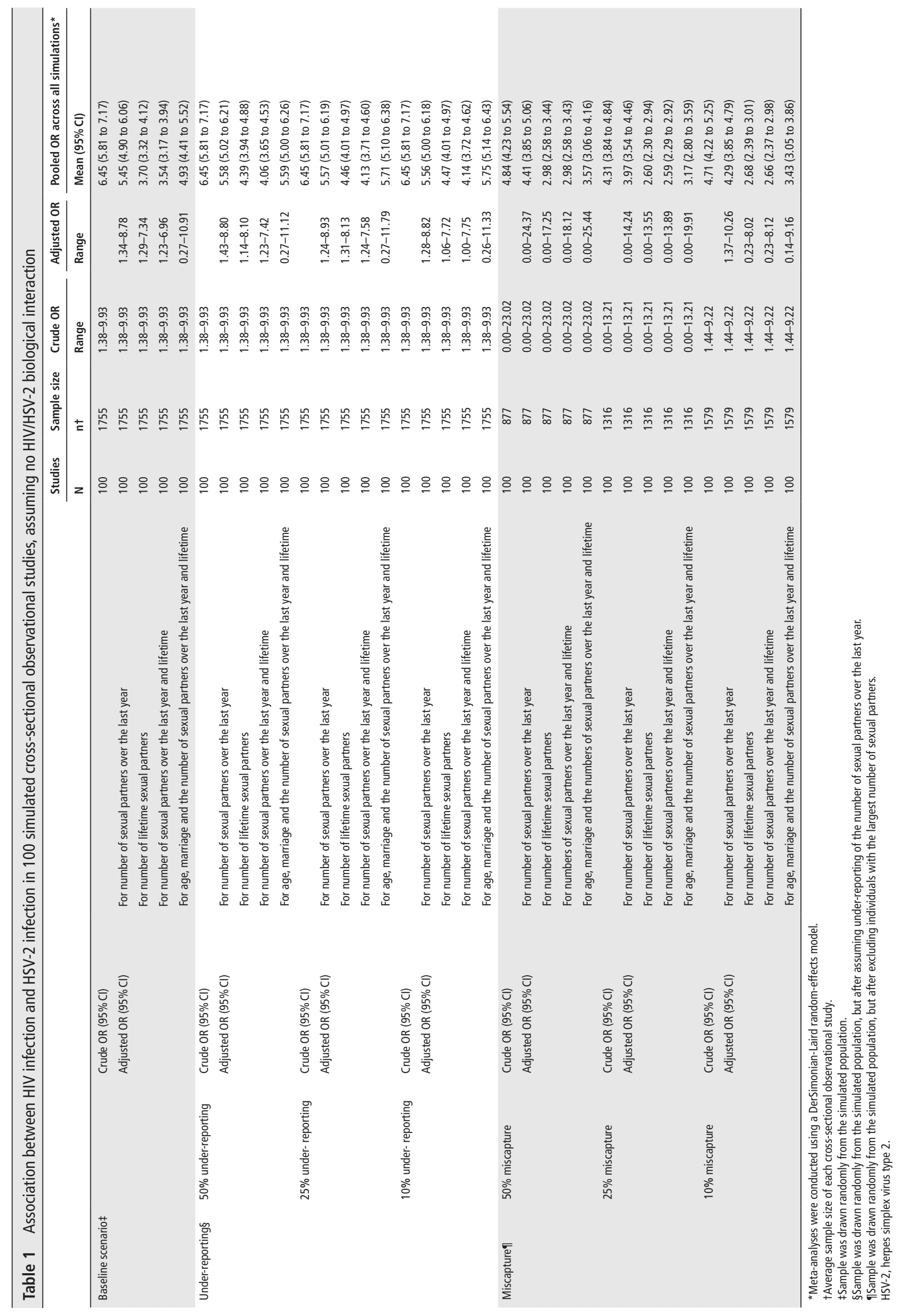




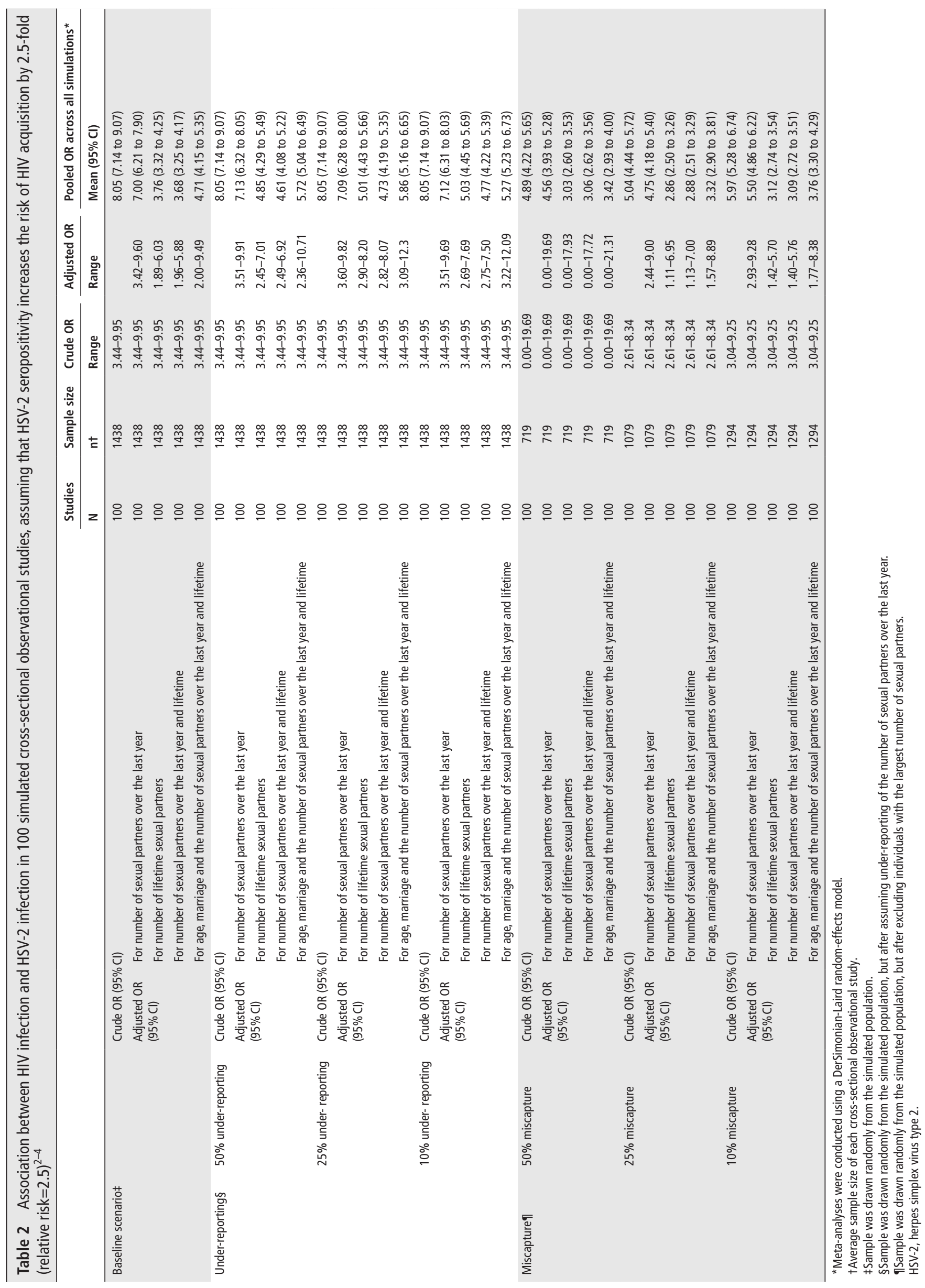


These findings highlight how HIV or HSV-2 exposure risk in a sexual network cannot be captured by simply numbers of partners, but depends on an array of network properties, including variance of number of partners, concurrency and clustering assortativeness. ${ }^{622}$

As expected, the ORs were larger in the positive-control analyses where HSV-2 seropositivity increased the risk of HIV acquisition (table 2). However, these ORs were not as large as one may have expected given the biological interaction. The adjustments for the sexual-behaviour confounding yielded also larger reductions in the ORs compared with the 'null' scenario of no interaction (table 2 vs table 1 ). These outcomes testify to the complexity of the HIV/HSV-2 overlapping epidemiology. AIDS mortality, for example, had a differential effect based on HSV-2 serostatus (with the higher risk of acquiring HIV for those HSV-2 seropositive), thereby lowering the values of the ORs.

Our results demonstrate how the ORs depend on complex interplays between the HIV/HSV-2 biological interaction, sexual behaviour and networking, HIV/HSV-2 background prevalence, demography, and AIDS mortality. This lack of a one-to-one correspondence between 'true' and observed ORs further increases the challenge of disentangling a biological interaction between HIV and HSV-2 from methodological biases.

This study has limitations. Our findings could conceivably be dependent on our model. While we studied this association across a diversity of sexual networks and HIV/HSV-2 epidemics, other factors, not accounted for here, may also affect the epidemiological observations, such as potential variation of this association by risk population, sex or biological route of exposure (eg, men who have sex with men versus heterosexual populations). Our results may not also explain the observed HIV/ HSV-2 synergy in serodiscordant couples. ${ }^{3}$ Having said so, the evidence from serodiscordant couples for an association appears inconclusive. ${ }^{3}$ Lastly, while our results cast doubt on an effect for HSV-2 on enhancing HIV acquisition, they do not strictly preclude an HSV-2 effect on enhancing HIV infectiousness.

In conclusion, can observational studies adequately control for confounding by sexual behaviour? Our findings demonstrate that it is challenging to assess the existence of an HIV/HSV-2 biological interaction in observational studies, as it is difficult (if not impossible) to fully control for the sexual-behaviour confounding using egocentric partners' data. Is the observed association consistent with an epidemiological synergy or confounding? The answer to this question remains unsettled, but our findings suggest that the empirically observed HIV/HSV-2 association appears to be more consistent with two infections sharing the same mode of transmission-HSV-2's effect on HIV acquisition may be weaker than thought, perhaps even absent.

\section{Handling editor Katy ME Turner}

Acknowledgements The authors gratefully acknowledge the fine support of Ms Adona Canlas in the preparation of this manuscript. The authors are also grateful for infrastructure support provided by the Biostatistics, Epidemiology, and Biomathematics Research Core at Weill Cornell Medicine-Qatar.

Contributors NN conceived this study. RO designed the study, developed the mathematical model and analysis plan, and performed the analyses. NN and $\amalg A-R$ contributed to study design and plan of analysis. LJA-R wrote the first draft of the article. All authors contributed to the interpretation of the results and drafting of the article.

Funding RO acknowledges the support of Precursory Research for Embryonic Science and Technology (PRESTO) grant number JPMJPR15E1 from Japan Science and Technology Agency (JST), and Japan Society for the Promotion of Science (JSPS), Grant-in-Aid for Young Scientists (B) 15K19217. This publication was made possible by NPRP grant number 5-752-3-177 from the Qatar National Research Fund (a member of Qatar Foundation). The findings achieved herein are solely the responsibility of the authors.

Competing interests None declared.

Provenance and peer review Not commissioned; externally peer reviewed.

Open access This is an open access article distributed in accordance with the Creative Commons Attribution Non Commercial (CC BY-NC 4.0) license, which permits others to distribute, remix, adapt, build upon this work non-commercially, and license their derivative works on different terms, provided the original work is properly cited and the use is non-commercial. See: http://creativecommons.org/ licenses/by-nc/4.0/

(c) Article author(s) (or their employer(s) unless otherwise stated in the text of the article) 2018. All rights reserved. No commercial use is permitted unless otherwise expressly granted.

\section{REFERENCES}

1 Abu-Raddad LJ, Magaret AS, Celum C, et al. Genital herpes has played a more important role than any other sexually transmitted infection in driving HIV prevalence in Africa. PLoS One 2008;3:e2230.

2 Wald A, Link K. Risk of human immunodeficiency virus infection in herpes simplex virus type 2-seropositive persons: a meta-analysis. J Infect Dis 2002;185:45-52.

3 Looker KJ, Elmes JAR, Gottlieb SL, et al. Effect of HSV-2 infection on subsequent HIV acquisition: an updated systematic review and meta-analysis. Lancet Infect Dis 2017.

4 Freeman EE, Weiss HA, Glynn JR, et al. Herpes simplex virus 2 infection increases HIV acquisition in men and women: systematic review and meta-analysis of longitudinal studies. AIDS 2006;20:73-83.

5 Freeman EE, Orroth KK, White RG, et al. Proportion of new HIV infections attributable to herpes simplex 2 increases over time: simulations of the changing role of sexually transmitted infections in sub-Saharan African HIV epidemics. Sex Transm Infect 2007;83():i17-i24.

6 Omori R, Abu-Raddad LJ. Sexual network drivers of HIV and herpes simplex virus type 2 transmission. AIDS 2017:31:1721-32

7 Day S, Ward H, Ison C, et al. Sexual networks: the integration of social and genetic data. Soc Sci Med 1998;47:1981-92.

8 Ghani AC, Swinton J, Garnett GP. The role of sexual partnership networks in the epidemiology of gonorrhea. Sex Transm Dis 1997;24:45-56.

9 Potterat JJ, Rothenberg RB, Muth SQ. Network structural dynamics and infectious disease propagation. Int J STD AIDS 1999;10:182-5.

10 Morris M. Network epidemiology: a handbook for survey design and data collection. Oxford University Press, 2004.

11 Morris M. Sexual networks and HIV. AIDS 1997;11 :S209-S16.

12 Neslon K, Williams CM E. Massachussets S, Infectious disease epidemiology: theory and practice. 2 nd. Sudbury, Massachussets: Jones and Bartlett Publishers, 2007.

13 Morris M, Kretzschmar M. Concurrent partnerships and the spread of HIV. AIDS 1997;11:641-8.

14 Watts $\mathrm{CH}$, May RM. The influence of concurrent partnerships on the dynamics of HIV/ AIDS. Math Biosci 1992;108:89-104.

15 Kretzschmar M, Morris M. Measures of concurrency in networks and the spread of infectious disease. Math Biosci 1996;133:165-95.

16 Lingappa JR, Baeten JM, Wald A, et al. Daily acyclovir for HIV-1 disease progression in people dually infected with HIV-1 and herpes simplex virus type 2: a randomised placebo-controlled trial. Lancet 2010:375:824-33.

17 Celum C, Wald A, Hughes J, et al. Effect of aciclovir on HIV-1 acquisition in herpes simplex virus 2 seropositive women and men who have sex with men: a randomised, double-blind, placebo-controlled trial. Lancet 2008;371:2109-19.

18 Watson-Jones D, Weiss HA, Rusizoka M, et al. Effect of herpes simplex suppression on incidence of HIV among women in Tanzania. N Engl J Med 2008;358:1560-71.

19 Tanton C, Abu-Raddad LJ, Weiss HA. Time to refocus on HSV interventions for HIV prevention? J Infect Dis 2011;204:1822-6.

20 Omori R, Chemaitelly H, Abu-Raddad LJ. Dynamics of non-cohabiting sex partnering in sub-Saharan Africa: a modelling study with implications for HIV transmission. Sex Transm Infect 2015;91:451-7.

21 MEASURE DHS. Demographic and Health Surveys. Calverton: ICF Macro. http://www. measuredhs.com/

22 Korenromp EL, de Vlas SJ, Nagelkerke NJ, et al. Estimating the magnitude of STD cofactor effects on HIV transmission: how well can it be done? Sex Transm Dis 2001;28:613-21. 